\title{
Coalition Structure Generation Utilizing Compact Characteristic Function Representations
}

\section{(Extended Abstract)}

\author{
Naoki Ohta \\ Kyushu University, Graduate School of ISEE \\ 744, Motooka, Nishi-ku \\ Fukuoka, Japan \\ ohta@agent.is.kyushu-u.ac.jp
}

\begin{abstract}
Forming effective coalitions is a major research challenge in AI and multi-agent systems. Coalition structure generation (CSG), which involves partitioning a set of agents into coalitions so that social surplus is maximized, is a central research topic due to its computational complexity. In this paper, we present new methods for CSG utilizing recently developed compact representation schemes for characteristic functions. We characterize the complexity of CSG under these representation schemes. In this context, the complexity is driven more by the number of "synergy coalition groups" than by the number of agents. Furthermore, we develop mixed integer programming formulations and show that an off-the-shelf optimization package can solve these problems quite efficiently.
\end{abstract}

\section{Categories and Subject Descriptors}

I.2.11 [Distributed Artificial Intelligence]: Multiagent systems; J.4 [Social and Behavioral Sciences]: Economics

\section{General Terms}

Theory, Economics

\section{Keywords}

Coalitional Game Theory, Optimization Problem

\section{INTRODUCTION}

Coalition formation is an important capability in automated negotiation among self-interested agents. Coalition structure generation (CSG) involves partitioning a set of agents into coalitions so that social surplus is maximized. This problem has become a popular research topic in AI and multi-agent systems. The CSG problem is equivalent to a complete set partition problem [7], and various algorithms for solving the CSG problem have been developed. Sandholm et al. propose an anytime algorithm with worst-case guarantees [6]. However, the worst-case time complexity is $O\left(n^{n}\right)$, where $n$ is the number of agents. On the other Cite as: Coalition Structure Generation Utilizing Compact Characteristic Function Representations (Short Paper), Naoki Ohta, Proc. of 8th Int. Conf. on Autonomous Agents and Multiagent Systems (AAMAS 2009), Decker, Sichman, Sierra and Castelfranchi (eds.), May, 10-15, 2009, Budapest, Hungary, pp. XXX-XXX.

Copyright (C) 2009, International Foundation for Autonomous Agents and Multiagent Systems (www.ifaamas.org). All rights reserved. hand, Dynamic Programming (DP) based algorithms [7, 3] are guaranteed to find an optimal solution in $O\left(3^{n}\right)$. Arguably, the state-of-the-art algorithm is the IP (integer partition) algorithm [4]. This is an anytime algorithm, which divides the search space into partitions based on integer partition, and performs branch \& bound search. Although the worst-case time complexity for obtaining an optimal solution is $O\left(n^{n}\right)$, in practice, IP is much faster than DP based algorithms. Furthermore, Rahwan et al. introduce an extension of the IP algorithm that utilizes DP for preprocessing [2].

As far as we are aware, all existing works on CSG assume that the characteristic function is represented implicitly, and we have oracle access to the function - that is, the value of a coalition (or a coalition structure as a whole) can be obtained using some procedure. This is because representing an arbitrary characteristic function explicitly requires $\Theta\left(2^{n}\right)$ numbers, which is prohibitive for large $n$. However, characteristic functions that appear in practice often display significant structure, and it is likely that such characteristic functions can be represented much more concisely. Indeed, recently, several new methods for representing characteristic functions have been developed [1]. These representation schemes capture characteristics of interactions among agents in a natural and concise manner, and can reduce the representation size significantly. Surprisingly, to our knowledge, these representation schemes have not yet been used for CSG; this is what we set out to do in this paper. We examine synergy coalition groups (SCGs) [1] which is one of these compact representation schemes. The optimal choice of a representation scheme depends on the application.

Quite interestingly, we find that there exists some common structure among these cases: in essence, the problem is to find a subset of "SCGs" that maximizes the sum of rule values under certain constraints. For each case, we show that solving the CSG problem is NP-hard, and the size of a problem instance is naturally measured by the number of "SCGs" rather than the number of agents. Also, we give a mixed integer programming (MIP) formulation that captures this structure. We show that an off-the-shelf optimization package (CPLEX) can solve the resulting MIP problem instances quite efficiently.

\section{MODEL}

Let $A=\{1,2, \ldots, n\}$ be the set of agents. A characteristic function $v: 2^{A} \rightarrow \Re$ assigns a value to each set of agents (coalition) $S \subseteq A$. We assume that each coalition's value 
is nonnegative. A coalition structure $C S$ is a partition of $A$, i.e., $C S=\left\{S_{1}, S_{2}, \ldots\right\}$ satisfies the following conditions: $\forall i, j(i \neq j), S_{i} \cap S_{j}=\emptyset, \bigcup_{S_{i} \in C S} S_{i}=A$. The value of a coalition structure $C S$, denoted as $V(C S)$, is calculated as follows: $V(C S)=\sum_{S_{i} \in C S} v\left(S_{i}\right)$. An optimal coalition structure $C S^{*}$ is a coalition structure that satisfies the following condition: $\forall C S, V\left(C S^{*}\right) \geq V(C S)$.

\section{CSG USING SCG}

Conitzer et al. introduce a concise representation of a characteristic function called a synergy coalition group (SCG) [1]. The main idea is to explicitly represent the value of a coalition only when there exists some positive synergy.

Definition 1 (SCG). An $S C G$ consists of a set of pairs of the form: $(S, v(S))$. For any coalition $S$, the value of the characteristic function is $v(S)=\max \left\{\sum_{S_{i} \in p_{S}} v\left(S_{i}\right): p_{S}\right.$ is a partition of $S$, i.e., all the $S_{i}$ are disjoint and $\bigcup_{S_{i} \in p_{S}} S_{i}=S$, and for all the $\left.S_{i},\left(S_{i}, v\left(S_{i}\right)\right) \in S C G\right\}$. To avoid senseless cases that have no feasible partitions, we require that $(\{a\}, 0) \in S C G$ whenever $\{a\}$ does not receive a value elsewhere in $S C G$.

Thus, if the value of a coalition $S$ is not given explicitly in $S C G$, it is calculated from the possible partitions of $S$. Using this original definition, we can represent only superadditive characteristic functions. But, if the characteristic function is super-additive, solving CSG becomes trivial: the grand coalition (the coalition of all agents) is optimal. To allow for characteristic functions that are not super-additive, we add the following requirement on the partition $p_{S}$.

- $\forall p_{S}^{\prime} \subseteq p_{S}$, where $\left|p_{S}^{\prime}\right| \geq 2,\left(\bigcup_{S_{i} \in p_{S}^{\prime}} S_{i}, v\left(\bigcup_{S_{i} \in p_{S}^{\prime}} S_{i}\right)\right)$ is not an element of $S C G$.

Example 1. Let there be five agents $a, b, c, d, e$ and let $S C G=\{(\{a\}, 0),(\{b\}, 0),(\{c\}, 1),(\{d\}, 2),(\{a, b\}, 3)$, $(\{a, b, c\}, 3)\}$. In this case, $v(\{a, b, c, d\})=v(\{a, b, c\})+$ $v(\{d\})=5$. We cannot use $v(\{a, b\})+v(\{c\})+v(\{d\})=6$, because $\{a, b\} \cup\{c\}=\{a, b, c\}$ appears in $S C G$.

The (modified) SCG can represent any characteristic function, including characteristic functions that are non-superadditive, or even non-monotone. This is because in the worst case, we can explicitly give the value of every coalition. Due to the additional condition, only these explicit values can then be used to calculate the characteristic function.

We show that when searching for $C S^{*}$, we need to consider only the coalitions that are explicitly described in $S C G$.

Theorem 1. There exists a coalition structure $C S$ for which $V(C S)=V\left(C S^{*}\right)$ and $\forall S \in C S,(S, v(S)) \in S C G$.

We omit the proofs in this report.

Due to Theorem 1, finding $C S^{*}$ is equivalent to a weighted set packing problem - equivalently, to the winner-determination problem in combinatorial auctions [5], where each agent is an item and each coalition described in $S C G$ is a bid.

Theorem 2. When the characteristic function is represented as an SCG, finding an optimal coalition structure is NP-hard. Moreover, unless $\mathcal{N} \mathcal{P}=\mathcal{Z} \mathcal{P} \mathcal{P}$, there exists no polynomial-time $O\left(|S C G|^{1-\epsilon}\right)$ approximation algorithm for any $\epsilon>0$.
Definition 2 (MIP formulation of CSG for SCG). The problem of finding $C S^{*}$ can be modeled as follows.

$$
\begin{array}{ll}
\max & \sum_{(S, v(S)) \in S C G} v(S) \cdot x(S) \\
\text { s.t. } & \forall a \in A, \sum_{S \ni a} x(S)=1, \\
& x(S) \in\{0,1\}
\end{array}
$$

$x(S)$ is 1 if $S$ is included in $C S^{*}, 0$ otherwise.

In this formulation (which corresponds to a standard winner determination formulation), the number of binary variables is equal to $|S C G|$, and the number of constraints is equal to the number of agents.

Our methods can solve a problem with 100 agents and 100 SCGs in less than 10 millisecond.

\section{CONCLUSION}

We showed that coalition structure generation can scale up significantly when the characteristic function is represented using recently developed a compact representation scheme which is called SCGs . For this case, we proved that the problem is NP-hard and inapproximable, and developed MIP formulations. Experimental results illustrated that while the state-of-the-art algorithm, which does not make use of compact representation, requires around 90 minutes to solve a problem with 27 agents, our methods can solve a problem with 100 agents and 100 SCGs in less than 10 millisecond. Future work includes developing anytime/approximation algorithms that utilize these representation schemes.

\section{REFERENCES}

[1] V. Conitzer and T. Sandholm. Complexity of constructing solutions in the core based on synergies among coalitions. Artificial Intelligence, 170(6):607-619, 2006.

[2] T. Rahwan and N. R. Jennings. Coalition structure generation: dynamic programming meets anytime optimisation. In Proc. of the 23rd Conf. on Artificial Intelligence (AAAI), pages 156-161, 2008.

[3] T. Rahwan and N. R. Jennings. An improved dynamic programming algorithm for coalition structure generation. In Proc. of the 7th Int. joint Conf. on Autonomous Agents and Multi-agent Systems (AAMAS), pages 1417-1420, 2008.

[4] T. Rahwan, S. D. Ramchurn, V. D. Dang, A. Giovannucci, and N. R. Jennings. Anytime optimal coalition structure generation. In Proc. of the 22nd Conf. on Artificial Intelligence (AAAI), pages 1184-1190, 2007.

[5] T. Sandholm. Algorithm for optimal winner determination in combinatorial auctions. Artificial Intelligence, 135(1-2):1-54, 2002.

[6] T. Sandholm, K. Larson, M. Andersson, O. Shehory, and F. Tohmé. Coalition structure generation with worst case guarantees. Artificial Intelligence, 111(1-2):209-238, 1999.

[7] D. Y. Yeh. A dynamic programming approach to the complete set partitioning problem. BIT Numerical Mathematics, 26(4):467-474, 1986. 